\title{
SMART - a computer program for modelling stellar atmospheres
}

\author{
A. Aret, A. Sapar, R. Poolamäe and L. Sapar \\ Tartu Observatory, 61602 Tõravere, Tartumaa, Estonia \\ email: aret@aai.ee
}

\begin{abstract}
Program SMART (Spectra and Model Atmospheres by Radiative Transfer) has been composed for modelling atmospheres and spectra of hot stars (O, B and A spectral classes) and studying different physical processes in them (Sapar \& Poolamäe 2003, Sapar et al. 2007). Lineblanketed models are computed assuming plane-parallel, static and horizontally homogeneous atmosphere in radiative, hydrostatic and local thermodynamic equilibrium. Main advantages of SMART are its shortness, simplicity, user friendliness and flexibility for study of different physical processes. SMART successfully runs on PC both under Windows and Linux.
\end{abstract}

Keywords. Radiative transfer, stars: atmospheres

\section{Main features of the program}

Model atmospheres are calculated iteratively varying only temperature and pressure dependency on column density. Flux constancy about $0.1-0.5 \%$ is achieved by about 10 iterations, using Kurucz ATLAS9 models as input. Number of atmospheric layers can be multiplied up if necessary. Line absorption has been completely taken into account with spectral resolution 300000 .

Programming language is Fortran 90, the program is compiled using Intel Fortran Compiler and runs both on Windows and Linux computers. Code is extensively commented on right-hand margin. Graphical interface (written in $\mathrm{C}++$ ) has been composed for visualizing results of calculation.

Radiative transfer has been calculated using integration by parts, yielding series of exponential integrals. The scattering processes are computed by simple $\Lambda$-iteration. Radiative transfer calculations give radiative flux $F_{\nu}(\lambda, \tau)$ in all layers of atmosphere.

Capabilities of program SMART include also computations of evolution of diffusive separation of isotopes in atmospheres of CP stars, relaxational formation of NLTE in line spectra, accelerations of clumps in stellar wind, computation of detailed spectral limb darkening and hence the spectra of rotating stars and non-irradiated eclipsing binaries. Pan-spectral method for determining element abundances from high-quality observed spectra have been developed and implemented as an extension to SMART.

The basic restriction is the assumption of plane-parallel, static and horizontally homogeneous atmosphere with no convection and no molecular absorption $\left(T_{\text {eff }}>9000 \mathrm{~K}\right)$. Present version assumes also LTE. NLTE calculations are not yet included into model computations. There are several simplifying assumptions reducing accuracy of modelling. Multiple light scattering is treated using simple $\Lambda$-iterations. A simplified treatment of Stark broadening of $\mathrm{H}$ and $\mathrm{He}$ lines has been used. Problems are also an instability of algorithms near Eddington limit and incompleteness of atomic data.

Typical running times on a PC with CPU $3.2 \mathrm{GHz}$ and 2 GB RAM are several hours for model atmosphere computation with spectral resolution 300000 and 64 layers of 
atmosphere. One time step in evolutionary computations of separation of mercury isotopes in atmospheres of CP stars with resolution 5000000 takes approximately 15 min.

\section{Special tasks}

SMART enables to compute evolution of chemical composition in atmospheres of CP stars due to diffusive separation of elements and isotopes driven by radiative acceleration, light-induced drift and gravity (Aret \& Sapar 2002, Sapar et al. 2005). Extensive highprecision line list and collision cross-sections are necessary. Currently the calculations have been made for mercury isotopes, similar calculations for calcium are in preparation.

Relaxational formation of NLTE in spectral lines can be calculated by rapidly converging iterations. Equilibrium quantum state populations of ion states are found from the equations of unbalanced statistical equilibrium treated as an relaxational initial value problem from LTE to NLTE populations.

Detailed spectral limb darkening has been computed for some model stellar atmospheres and used thereafter for finding spectra of rotating stars and non-irradiated eclipsing binaries. Codes also accounting for stellar surface distortion and gravitational darkening are currently prepared.

To enlighten the problem of stellar wind triggering in stellar atmospheres, the radiative acceleration of moving clumps with Doppler shifted spectral lines has been studied and found to give hopeful results.

Pan-spectral method for determining element abundances (Sapar et al. 2008) aimed for the automatic processing of high-quality stellar spectra has been elaborated. The method is based on weighted cumulative line-widths $Q_{\lambda}$ defined as

$$
Q_{\lambda}=\int_{\lambda_{0}}^{\lambda}\left|\frac{d R_{\lambda}}{d Z}\right|\left(1-R_{\lambda}\right) d \lambda,
$$

where $R_{\lambda}$ is the residual flux (intensity) and $Z=\log \left(N_{\text {elem }} / N_{\text {tot }}\right)$ is the abundance of studied element or isotope. The derivative of residual flux $R_{\lambda}$ with respect to abundance $Z$ automatically excludes spectral regions insensitive to changes of the abundance of studied element and gives a large contribution in the most sensitive regions, i.e. in the centres of non-saturated lines and in the steep wings of strong lines of the element. Best fit of quantities $Q_{\lambda}$ found from synthetic and observed spectra gives final abundance taking duly into account all lines of studied element including blended ones. Abundances can be found simultaneously for many elements. The method can also be used to find corrections of effective temperature and gravity.

\section{Acknowledgements}

We are grateful to Estonian Science Foundation for financial support by grant ETF 6105 .

\section{References}

Sapar A. \& Poolamäe R. 2003, ASP Conf. Series, 288, 95

Sapar A., Poolamäe R., Sapar L., \& Aret, A. 2007, Spectroscopic Methods in Modern Astrophysics, INASAN, Moscow, 236 (in Russian)

Aret A. \& Sapar A. 2002, Astr. Nachr., 323, 1, 21

Sapar A., Aret A., \& Poolamäe R. 2005, EAS Publ. Series, 17, 341

Sapar A., Aret A., Sapar L., \& Poolamäe R. 2008, Precision Spectroscopy in Astrophysics: ESO Astrophysics Symposia, Springer Verlag, 145 